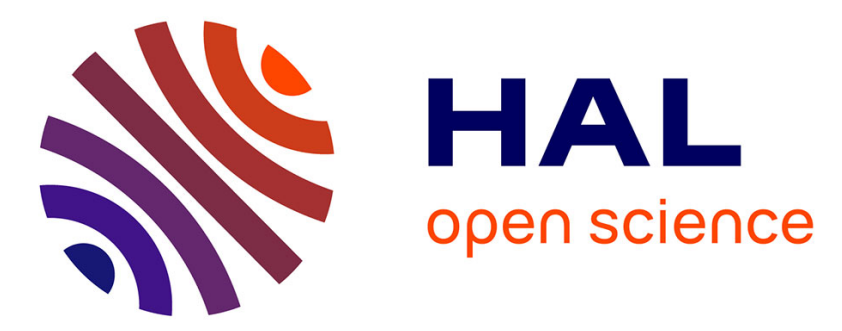

\title{
Singlet excited state dynamics of uracil and thymine derivatives: A femtosecond fluorescence upconversion study in acetonitrile
}

\author{
T. Gustavsson, N. Sarkar, E. Lazzarotto, D. Markovitsi, R. Improta
}

\section{- To cite this version:}

T. Gustavsson, N. Sarkar, E. Lazzarotto, D. Markovitsi, R. Improta. Singlet excited state dynamics of uracil and thymine derivatives: A femtosecond fluorescence upconversion study in acetonitrile. Chemical Physics Letters, 2006, 429, pp.551-557. 10.1016/j.cplett.2006.08.058 . hal-00098348

\section{HAL Id: hal-00098348 \\ https://hal.science/hal-00098348}

Submitted on 25 Sep 2006

HAL is a multi-disciplinary open access archive for the deposit and dissemination of scientific research documents, whether they are published or not. The documents may come from teaching and research institutions in France or abroad, or from public or private research centers.
L'archive ouverte pluridisciplinaire $\mathbf{H A L}$, est destinée au dépôt et à la diffusion de documents scientifiques de niveau recherche, publiés ou non, émanant des établissements d'enseignement et de recherche français ou étrangers, des laboratoires publics ou privés. 


\title{
Singlet excited state dynamics of uracil and thymine derivatives. A femtosecond fluorescence upconversion study in acetonitrile
}

\author{
Thomas Gustavsson*, Nilmoni Sarkar ${ }^{1}$, Elodie Lazzarotto, Dimitra Markovitsi
}

Laboratoire Francis Perrin, CEA/DSM/DRECAM/SPAM - CNRS URA 2453,

CEA Saclay, F-91191 Gif-sur-Yvette, France

Roberto Improta

\#Dipartimento di Chimica, Universita Federico II, Complesso Universitario Monte S. Angelo, Via Cintia, I-80126 Napoli, Italy and Istituto Biostrutture e Bioimmagini/CNR, V.

Mezzocannone 6-80134 Napoli, Italy

\section{AUTHOR EMAIL ADDRESS *: thomas.gustavsson@cea.fr}

\begin{abstract}
The excited state properties of uracil, thymine and four analogous uracil compounds have been studied in acetonitrile by steady-state and time-resolved spectroscopy. The excited state lifetimes were measured using femtosecond UV fluorescence upconversion. The excited state lifetimes of uracil and its 1- and 3-methyl substituted derivatives are well described by one ultrafast ( $\leq 100 \mathrm{fs}$ ) component. 5-substituted compounds show a more complex behavior, exhibiting longer excited state lifetimes and biexponential fluorescence decays. These longer decays are substantially faster in acetonitrile than in aqueous solution showing that the excited state deactivation mechanism is in part governed by the solvent.
\end{abstract}

${ }^{1}$ Permanent address : Department of Chemistry, Indian Institute of Technology, Kharagpur, PIN 721 302, WB, India. 


\section{Introduction}

There is currently a renewed interest in characterizing optically excited states of DNA and of its constituents [1] and to determine the relevance of these states in the complex mechanisms of DNA photodamage. The extremely short lifetimes of these directly photoexcited states have for long hampered detailed studies, making it difficult to understand how the injected excess energy evolves in time. Only in the last few years, due to very recent advances of ultrafast time-resolved spectroscopic techniques, has it been possible to measure the excited-state decays of nucleobases (adenine, thymine, cytosine, guanine) and of the corresponding nucleosides and nucleotides with a sufficiently high time-resolution to see that they occur on a sub-picosecond time scale.

Kohler and coworkers have successfully used femtosecond transient absorption spectroscopy to study the DNA building blocks [2-9], showing that an induced absorption in the red spectral region is due to the directly photoexcited state. In parallel, Zewail and coworkers [10], and Pancur et al. [11] have used fluorescence upconversion to measure nucleoside and nucleotide excited state lifetimes. These two complementary methods agree on the ultrafast decay times of the first singlet excited state.

At the same time we have, in a series of papers, studied the fluorescent state of the monomeric DNA constituents by femtosecond fluorescence upconversion [12-16]. In particular, a recent exhaustive study of several uracils in aqueous solution [17] shows that most of these compounds are deactivated faster than our time-resolution (100 fs). Only 5substituted compounds (among them thymine) show a more complex behavior, exhibiting longer excited state lifetimes and biexponential fluorescence decays. A parallel theoretical analysis confirmed the role, both statical and dynamical, of the 5-substituent in the deactivation process of uracils.

In general, the mechanism responsible for the ultrafast non-radiative deactivation (internal conversion) has not yet been completely assessed, even if several theoretical studies point towards the existence of near barrier-less paths, implying important ring deformation, leading from the excited state through a conical intersection to the ground state both in uracil [17-21], cytosine [8,22-24] and adenine [19,25-29]. Even if the proposed deactivation mechanism is not identical for the three bases (therefore one should not think of it in terms of a general mechanism, common for all DNA bases), the present picture is thus that the ultrafast decays observed for the various bases are due to purely intramolecular mechanisms, little or not affected by the solvent. However, gas phase studies have shown that much longer lived 
states, having nanosecond lifetimes, exist in vacuum [30]. The environment may therefore play an important role, directly or indirectly by tuning the electronic states and therefore change the energetics governing the formation of conical intersections. Hydrogen bonding cannot be excluded, all the nucleobases have more or less pronounced $\mathrm{H}$-bond acceptor or donor character and in many cases multiple tautomeric forms coexist.

Up to this date, nearly all ultrafast studies have been performed in aqueous solution. Only a few exceptions can be found: in particular two studies have been done in acetonitrile, a polar but aprotic solvent, thus excluding the formation of solute/solvent hydrogen bonds. Häupl et al. measured the lifetimes of various nucleobases in water, ethanol and acetonitrile using a streak camera with picosecond time-resolution [31]. Interestingly, they measured longer fluorescence lifetimes of thymine in acetonitrile (2.4 ps) and in ethanol (2.2 ps), than in water (1.5 ps). On the other hand they observed a significant increase of the fluorescence lifetime of adenine in ethanol (16.2 ps) with respect to that in water (8.5 ps) or acetonitrile (8.7 ps). Cohen et al. investigated the solvent effect on ultrafast excited-state dynamics of adenine and monomethylated adenines in water, $\mathrm{D}_{2} \mathrm{O}$ and acetonitrile by transient absorption [4]. For adenine and 9-methyladenine they observed slight increases in lifetimes when going from water to acetonitrile, while for 7-methyladenine a non-negligible decrease, from 4.23 to $3.3 \mathrm{ps}$, was observed. In summary, the situation is not clear and the role of the solvent in the ultrafast non-radiative excited state deactivation of nucleobases is neither clearly identified nor well understood. The solvent effect on the excited state relaxation of nucleobases is said to be 'modest' [1].

In a recent study of 5-fluorouracil we have instead shown that the excited state decay is significantly faster in acetonitrile than in water. By combining time-resolved fluorescence spectroscopy and TD-DFT calculations on the excited state we suggested that the relative ordering of the first excited $\pi \pi^{*}$ and $n \pi^{*}$ states depends strongly on the solvent [32]. Indeed, contrary to the situation in aqueous solution, in acetonitrile these states become neardegenerated, opening up an additional decay channel for the optically bright $\pi \pi^{*}$ state, in accordance with an observed faster decay.

As a further step towards the understanding of solvent effect on the excited state behavior of nucleobases we have thus extended our studies in acetonitrile solution to five other uracils (including uracil and thymine), comparing the findings with those obtained in aqueous solution. 


\section{Experimental}

All compounds; uracil (U), thymine (5-methyluracil) (T), 6-methyluracil (6MU), 1,3dimethyluracil (1,3DMU), 5-fluorouracil (5FU) and 5-trifluoromethyluracil (TFT), see Chart 1, were purchased from Sigma Aldrich and acetonitrile (UV spectroscopic grade) from Merck. All products were used without further purification.

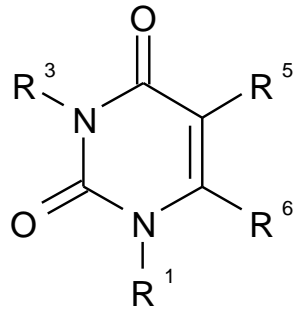

\begin{tabular}{l||c|c|c|c} 
& $\mathrm{R}^{1}$ & $\mathrm{R}^{3}$ & $\mathrm{R}^{5}$ & $\mathrm{R}^{6}$ \\
\hline \hline $\mathrm{U}$ & $\mathrm{H}$ & $\mathrm{H}$ & $\mathrm{H}$ & $\mathrm{H}$ \\
T & $\mathrm{H}$ & $\mathrm{H}$ & $\mathrm{Me}$ & $\mathrm{H}$ \\
6MU & $\mathrm{H}$ & $\mathrm{H}$ & $\mathrm{H}$ & $\mathrm{Me}$ \\
1,3DMU & $\mathrm{Me}$ & $\mathrm{Me}$ & $\mathrm{H}$ & $\mathrm{H}$ \\
5FU & $\mathrm{H}$ & $\mathrm{H}$ & $\mathrm{F}$ & $\mathrm{H}$ \\
TFT & $\mathrm{H}$ & $\mathrm{H}$ & $\mathrm{CF}_{3}$ & $\mathrm{H}$
\end{tabular}

Chart 1. The schematic structure of the substituted uracils studied in the present work, where $R^{1}, R^{2}, R^{3}$ and $R^{4}$ denote the different substituents corresponding to the table.

Absorption spectra were recorded with a Perkin-Elmer Lamda 900 spectrophotometer using $1 \mathrm{~mm}, 2 \mathrm{~mm}$ and $1 \mathrm{~cm}$ quartz cells (QZS). Fluorescence spectra were recorded with a SPEX Fluorolog-2 spectrofluorimeter. The light source was a 450 Watt arc Xenon lamp.

For the fluorescence measurements, a $1 \mathrm{~cm} \mathrm{x} 1 \mathrm{~cm}$ quartz cell was used for all compounds except uracil. To measure the fluorescence spectra of uracil we have used $0.2 \times 1 \mathrm{~cm}$ quartz cell. Acetonitrile has an intrinsic longlived (as controlled by separate TCSPC measurements) emission impurity on the ppb level which emits around $365 \mathrm{~nm}$. This signal is not negligible compared to the fluorescence of the uracil derivatives. Therefore, the neat acetonitrile fluorescence must be subtracted from all spectra. In this way the weak continuum was almost removed but neither the Raman line nor the weak continuum at the red end of the emission spectra could be completely eliminated.

Both the absorption and fluorescence spectra of the various uracils differ strongly in position and shape. As a consequence, in order to compare more easily and precisely the spectral properties of the different compounds examined, both absorption and fluorescence spectra were put on a frequency scale. Fluorescence spectra were scaled by a $\lambda^{2}$ factor and normalized. Both the absorption and fluorescence spectra were fitted with a simplified lognormal function as described in our earlier publication to evaluate the peak frequency and full width half maxima [17]. 
The femtosecond fluorescence upconversion setup has been described earlier [12]. The excitation source is the third harmonic of a mode-locked Ti-sapphire laser. The $267 \mathrm{~nm}$ pulses are generated in a home-made frequency-tripler using two $0.5 \mathrm{~mm}$ type I BBO crystals. Typically, the average excitation power used at $267 \mathrm{~nm}$ was $40 \mathrm{~mW}$. The fluorescence from the sample is collected by parabolic mirrors and mixed with the residual fundamental in a $0.5 \mathrm{~mm}$ type I BBO crystal in order to generate the sum-frequency. The sum-frequency light is spectrally filtered in a monochromator and detected by a photomultiplier in single-photon counting mode. The spectral resolution of the monochromator at the detection wavelength $(223-288 \mathrm{~nm})$ was set to $8 \mathrm{~nm}$. Parallel $\left(I_{\text {par }}(t)\right)$ and perpendicular $\left(I_{\text {perp }}(t)\right)$ excitation/detection configurations were realized by controlling the polarization of the exciting beam with a zero-order half-wave plate.

Fluorescence decays were recorded at 330 and $350 \mathrm{~nm}$. Temporal scans were made with 33.3 fs steps in both parallel and perpendicular configurations. The fwhm value of a Gaussian apparatus function is about $350 \mathrm{fs}$ at $330 \mathrm{~nm}$ and decreases to about $300 \mathrm{fs}$ at $360 \mathrm{~nm}$ as expected from the GVD mismatch between the fluorescence and the fundamental in the sumfrequency crystal. We judge that the time resolution of our setup is better than $100 \mathrm{fs}$ after deconvolution, depending on the signal-to-noise ratio.

All upconversion measurements were performed at room temperature $\left(20 \pm 1^{\circ} \mathrm{C}\right)$ under aerated conditions. Solutions $\left(\approx 2.5 \times 10^{-3} \mathrm{~mol} / \mathrm{dm}^{-3}\right)$ were kept flowing through a $0.4 \mathrm{~mm}$ quartz cell, which itself was kept in continuous motion perpendicular to the excitation beam. The pulse intensity cannot be measured precisely within the excitation volume but we estimate it to $0.2 \pm 0.1 \mathrm{GW} / \mathrm{cm}^{2}$ for a $40 \mathrm{~mW}$ output from the tripler unit (assuming a 40 micron diameter of the focused beam).

All solutions were slightly discolored after the upconversion experiments, the fluorinated compounds less than the others. For all compounds several (tens) decays were recorded and averaged. No systematic variations were observed from one decay to another so we judge that the eventual photoproducts do not perturb the decays on the femtosecond timescale, which can be taken as a minimum condition for the validity of the data [33]. Upconversion recordings with neat acetonitrile did not produce any measurable signal, so we judge that the fluorescence decays recorded for the various uracils are not influenced by the intrinsic longlived emission from acetonitrile mentioned above. 


\section{Results}

\section{Steady-state absorption and fluorescence spectra}

Steady-state absorption and fluorescence spectra of uracil, thymine, 6-methyluracil, 1,3dimethyluracil, 5-fluorouracil and 5-trifluorothymine in acetonitrile are shown in Figure 1. Characteristic parameters of the first absorption and fluorescence bands of the six uracils are given in Table 1.

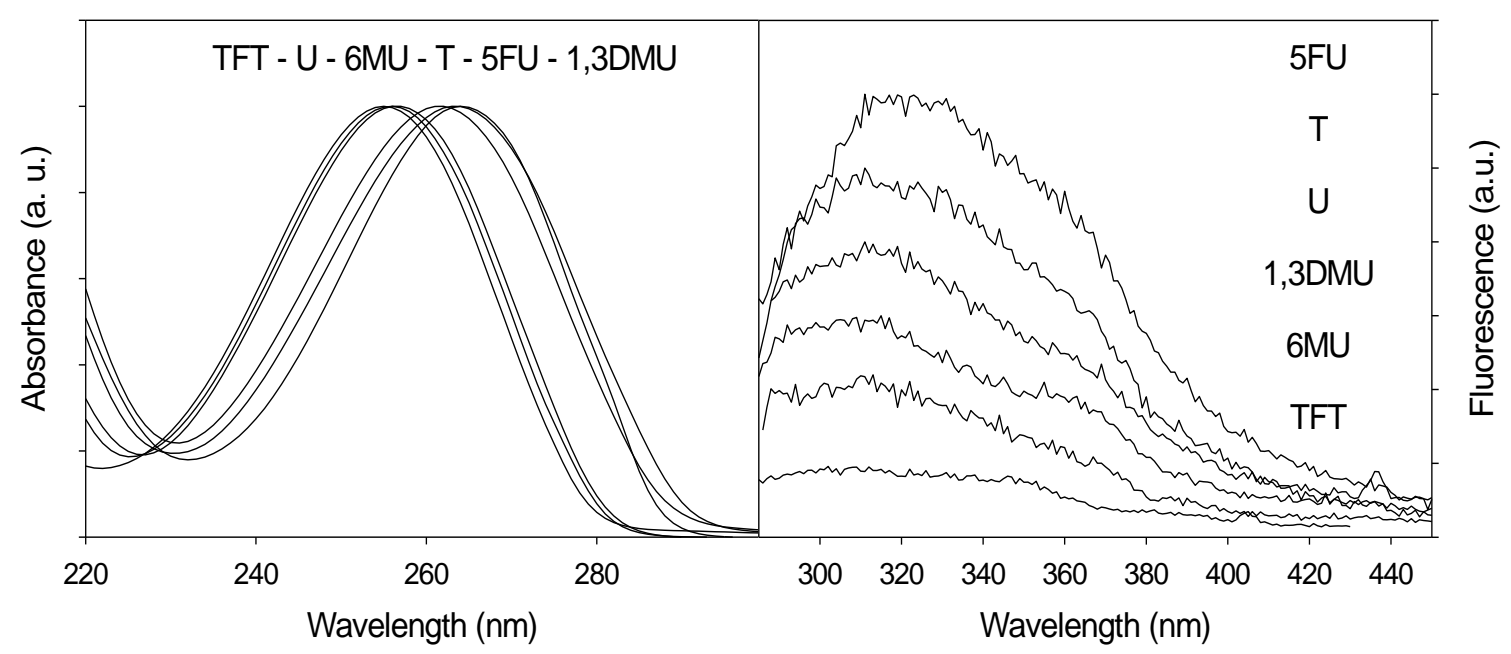

Figure 1. Steady-state absorption (left panel) and fluorescence (right panel) spectra of six uracils in roomtemperature acetonitrile solutions; uracil (U), 6-methyluracil (6MU), 1,3-dimethyluracil (1,3DMU), 5-trifluoromethyluracil (TFT), thymine (T) and 5-fluorouracil (5FU). Absorption spectra have been normalized. In order to increase the readability of the figure, fluorescence spectra are depicted in the same order as the estimated fluorescence quantum yield.

\section{Steady state absorption spectra:}

Uracil, 6-methyluracil and 5-trifluorothymine exhibit absorption maxima around $255 \mathrm{~nm}$, whereas the absorption maxima of thymine, 1,3-dimethyluracil and 5-fluorouracil are above $260 \mathrm{~nm}$. All the absorption maxima, corresponding to a bright $\pi / \pi^{*}$ state $[17,20,32]$, are blue shifted in acetonitrile compared to aqueous solution. The shift ranges from $\approx 100 \mathrm{~cm}^{-1}$ in the case of $6 \mathrm{MU}$ to $\approx 500 \mathrm{~cm}^{-1}$ for uracil (see Table 1 ). These results confirm $[17,32]$ that the relative stability of the lowest energy $\pi / \pi^{*}$ state increases with the polarity of the embedding medium (the dielectric constants of water and acetonitrile are about 80 and 40, respectively). We recall indeed that in the gas phase the absorption maxima of uracil and 1,3DMU fall at 244 and $256 \mathrm{~nm}$, respectively [34]. Only a few studies of the spectral properties of uracil in non- aqueous solution have been reported. Clark and Tinoco Jr. reported absorption spectra of 1,3-dimethyluracil in trimethyl phosphate $(264 \mathrm{~nm})$ and methylcyclohexane $(262 \mathrm{~nm})$ and 
uracil in trimethyl phosphate $(258 \mathrm{~nm})$ [35]. Trimethylphosphate and methylcyclohexane are both low-polarity and non-hydrogen bonding solvents. These values compare well to our observations, 1,3-dimethyluracil in water $(266 \mathrm{~nm})$ and acetonitrile $(264 \mathrm{~nm})$, uracil in water $(259 \mathrm{~nm})$ and acetonitrile $(256 \mathrm{~nm})$.

The substituent effect on the absorption spectra is the same in acetonitrile and in water. Methyl substitution in position 1 or 5 produces a noticeable red shift of the absorption maximum compared to uracil [17], whereas methyl substitution in position 3 or 6 does not have any noticeable effect.

More precisely, in acetonitrile the absorption maximum of thymine, 1,3-dimethyluracil, and 5-fluorouracil are red shifted compared to uracil by about $900 \mathrm{~cm}^{-1}, 1200 \mathrm{~cm}^{-1}$, and $1200 \mathrm{~cm}^{-1}$, respectively. Interestingly, the absorption maximum of 5-trifluorothymine is slightly blue-shifted compared to uracil in acetonitrile.

\section{Steady state fluorescence spectra:}

Steady-state fluorescence spectra of the uracils in acetonitrile solution at room temperature obtained for excitation at $255 \mathrm{~nm}$ (except for TFT that was excited at $240 \mathrm{~nm}$ ) were analyzed as described above and resulting spectral parameters are reported in Table 1. As in aqueous solution [17], all the compounds examined exhibit a rather large Stokes shift (ranging from about $6800 \mathrm{~cm}^{-1}$ for 1,3-dimethyluracil to more than $8200 \mathrm{~cm}^{-1}$ for uracil), suggesting significant changes of the excited state geometry also in acetonitrile. Some remarkable features concerning the dependence of the Stokes shift on the substituent and the solvent can be highlighted. For example, while in water the Stokes Shift of thymine and 5-fluorouracil is significantly larger than that of uracil, the opposite is found in acetonitrile. Actually in this latter solvent uracil exhibits the largest Stokes shift among the compounds examined, whereas in water solution 5-fluorouracil exhibits the largest Stokes Shift.

As found in the absorption spectra, in acetonitrile the fluorescence maximum of thymine and, especially, of 5-fluorouracil are red-shifted with respect to that of uracil. On the contrary the fluorescence peak maxima of 6-methyluracil, 1,3-dimethyluracil and 5-trifluorothymine are very close and slightly blue-shifted with respect to uracil. This trend is similar to that found in water. In this latter solvent, however, the energy of the fluorescence peaks is more dispersed. For example, the energy difference between the fluorescence peak of 5-fluoruracil and uracil is $1.900 \mathrm{~cm}^{-1}$ in water and $900 \mathrm{~cm}^{-1}$ in acetonitrile. 
Table 1. Characteristic parameters of the first absorption and fluorescence bands of uracil and its derivatives in $\mathrm{CH}_{3} \mathrm{CN}$ (values in parentheses refer to $\mathrm{H}_{2} \mathrm{O}$ data taken from ref. [17]). The peak frequency $v_{\text {max }}$, the peak wavelength $\lambda_{\max }$, the width $\delta v(f w h m)$ and the Stokes shift $\Delta v$ (peak absorption minus peak fluorescence).

\begin{tabular}{c|cccc|ccc|c}
\hline \multirow{2}{*}{ Compound } & \multicolumn{4}{|c|}{ absorption } & \multicolumn{3}{c|}{ fluorescence } & Stokes shift \\
\cline { 2 - 9 } & $v_{\max }$ & $\lambda$ & $\delta v$ & $v_{\max }$ & $\lambda_{\max }$ & $\delta v$ & $\Delta v$ \\
\cline { 2 - 9 } & $\left(\mathrm{cm}^{-1}\right) \cdot 10^{3}$ & $(\mathrm{~nm})$ & $\left(\mathrm{cm}^{-1}\right) \cdot 10^{3}$ & $\left(\mathrm{~cm}^{-1}\right) \cdot 10^{3}$ & $(\mathrm{~nm})$ & $\left(\mathrm{cm}^{-1}\right) \cdot 10^{3}$ & $\left(\mathrm{~cm}^{-1}\right) \cdot 10^{3}$ \\
\hline uracil & $39.1(38.6)$ & 256 & 4.8 & $30.9(31.3)$ & 311 & 8.5 & $8.2(7.3)$ \\
\hline 6-methyluracil & $39.0(38.9)$ & 256 & 4.7 & $31.5(31.1)$ & 308 & 8.3 & $7.5(7.8)$ \\
\hline 1,3-dimethyluracil & $37.9(37.6)$ & 264 & 4.4 & $31.1(31.5)$ & 308 & 9.1 & $6.8(6.1)$ \\
\hline 5-trifluorothymine & $39.2(38.9)$ & 255 & 4.6 & $31.3(31.5)$ & 307 & 9.8 & $7.9(7.4)$ \\
\hline thymine & $38.2(37.8)$ & 261 & 4.4 & $30.6(29.9)$ & 315 & 8.6 & $7.6(7.8)$ \\
\hline 5-fluorouracil & $37.9(37.6)$ & 264 & 4.8 & $30.0(29.4)$ & 322 & 7.9 & $7.9(8.2)$ \\
\hline
\end{tabular}

\section{Fluorescence decays}

Fluorescence decays were recorded for $\sim 2.5 \times 10^{-3} \mathrm{~mol} / \mathrm{dm}^{-3}$ aqueous solutions at 330 $\mathrm{nm}$ after $267 \mathrm{~nm}$ excitation. Total fluorescence kinetics $F(t)$ shown below were constructed from the parallel and perpendicular signals $\left(I_{p a r}(t)\right.$ and $\left.I_{\text {perp }}(t)\right)$ according to the equation

$$
F(t)=I_{p a r}(t)+2 G I_{p e r p}(t)
$$

where $\mathrm{G}$ is the ratio of the sensitivity of the detection system to fluorescence polarized parallel and perpendicular to the (vertical) excitation light. In our case G was measured to 0.95 .

The resulting decay curves for uracil, 6-methyluracil, 1,3-dimethyluracil, 5methyluracil (thymine), 5-fluorouracil and 5-trifluorothymine are shown in Figure 2. Also displayed in this figure is the $330 \mathrm{fs}$ (fwhm) Gaussian apparatus function.

The fluorescence decays can be classified into two groups, the first consisting of uracil, 6-methyluracil, 1,3-dimethyluracil and 5-trifluorothymine, showing an ultrafast decay basically limited by the response function, and a second "group" constituted by thymine and 5-fluorouracil characterized by a much longer decay.

In order to quantify these observations, we performed a merged nonlinear fitting/deconvolution process using a model function constructed by the convolution of a model decay function (the impulse response function), $i(t)$, and a Gaussian instrument response function, $G(t)$. The resulting fitting function is then written as $I(t) \propto i(t) \otimes G(t)$. 


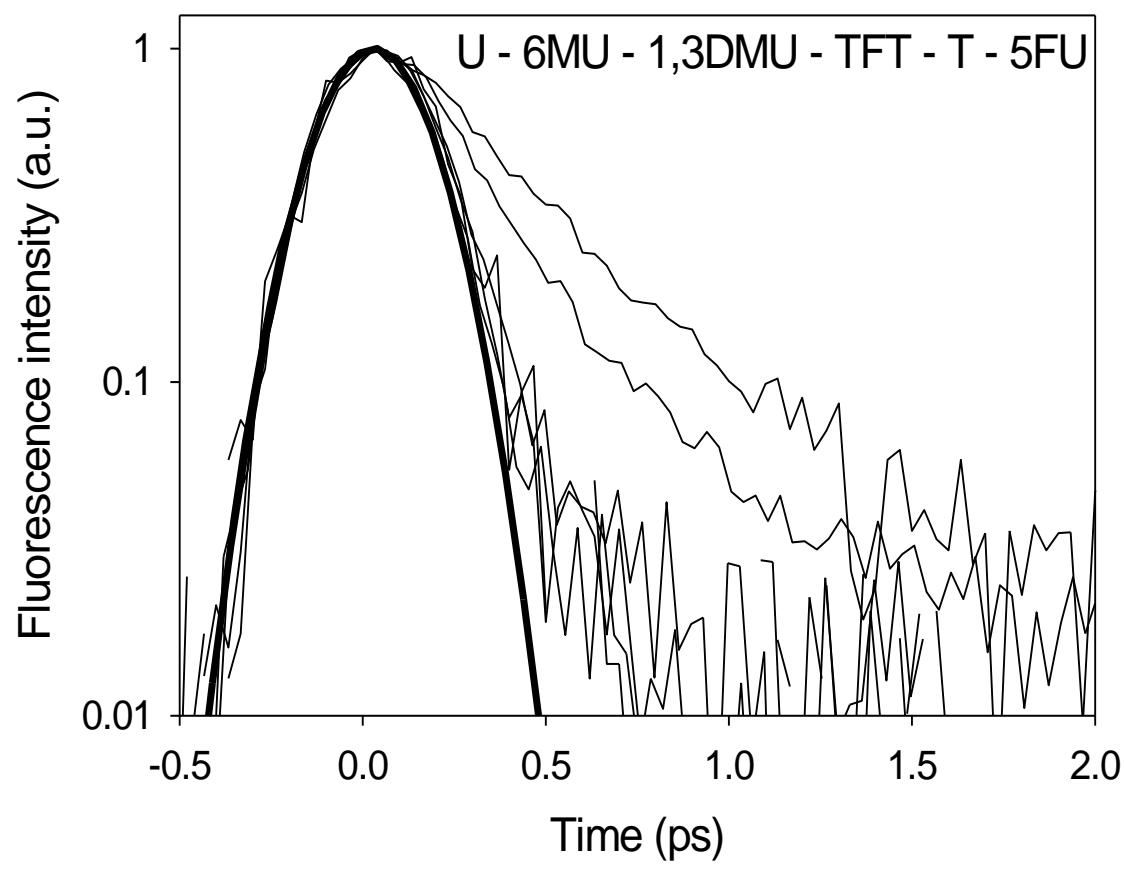

Figure 2. Fluorescence decays on a semi-log scale at $330 \mathrm{~nm}$ after excitation at $267 \mathrm{~nm}$ of the six uracils in room-temperature acetonitrile solutions $\left(\sim 2.5 \times 10^{-3} \mathrm{~mol} / \mathrm{dm}^{-3}\right)$; (in increasing order) uracil, 6-methyluracil, 1,3dimethyluracil, 5-trifluorothymine, thymine and 5-fluorouracil. Also shown (thick solid line) is the $330 \mathrm{fs}$ ( $f w h m$ ) Gaussian apparatus function.

The fluorescence decays of the first group are well characterized by representing $i(t)$ by a mono-exponential function having a $\approx 100 \mathrm{fs}$ lifetime. However this time constant should only be taken as "upper" limit, due to our time-resolution (judged to be $100 \mathrm{fs}$ after deconvolution). The real time-constants could be much faster.

For thymine and 5-fluorouracil, on the other hand, a bi-exponential function was used for the parameter estimation and the fluorescence decays were found to be well described by one ultrafast component varying between 80 and 300 fs and a second much slower component, see Table 2 .

Table 2. Measured characteristic times ( $f$ s) of the fluorescence decays of uracil and its derivatives in $\mathrm{CH}_{3} \mathrm{CN}$.Values in parentheses correspond to measures limited by the time resolution.

\begin{tabular}{l|c|c|c|c}
\hline Compound & $\mathbf{a}_{\mathbf{1}}$ & $\tau_{\mathbf{1}}(\mathbf{f s})$ & $\tau_{\mathbf{2}}(\mathbf{f s})$ & $\langle\tau\rangle(\mathbf{f s})$ \\
\hline uracil & 1 & $(100)$ & & \\
\hline 6-methyluracil & 1 & $(100)$ & & \\
\hline 1,3-dimethyluracil & 1 & $(100)$ & & \\
\hline 5-trifluorothymine & 1 & $(100)$ & & \\
\hline thymine & 0.95 & 190 & 1100 & 235 \\
\hline 5-fluorouracil & 0.88 & 300 & 1012 & 392 \\
\hline
\end{tabular}


It is thus worth underlining that the only compounds showing fluorescence decays substantially longer than the apparatus function are thymine and 5-fluorouracil. These two compounds showed slower decays than uracil also in water.

In order to clearly show the difference between the fluorescence decays of thymine in water and in acetonitrile, they are compared separately in Figure 3. The noise level is slightly higher in acetonitrile since most uracils are less soluble in this solvent, causing a weaker signal. Interestingly, a careful inspection of Figure 3 indicates that the fluorescence decay of thymine in acetonitrile is highly non-exponential, leveling out at $2 \mathrm{ps}$. The slow component, $\tau_{2}$ $=1.1 \mathrm{ps}$, is longer than the corresponding one in water, $\tau_{2}=0.4 \mathrm{ps}$, even though the average lifetime, $\langle\tau\rangle$, is faster in acetonitrile than in water, 235 vs. 388 fs. Confirming the results already reported for 5-fluorouracil [32], the excited state decay of thymine is thus faster in acetonitrile than in water. An analogous and interesting difference between these two solvents concerns 5-trifluorothymine that shows a bi-exponential fluorescence decay in water but an ultrafast mono-exponential decay in acetonitrile.

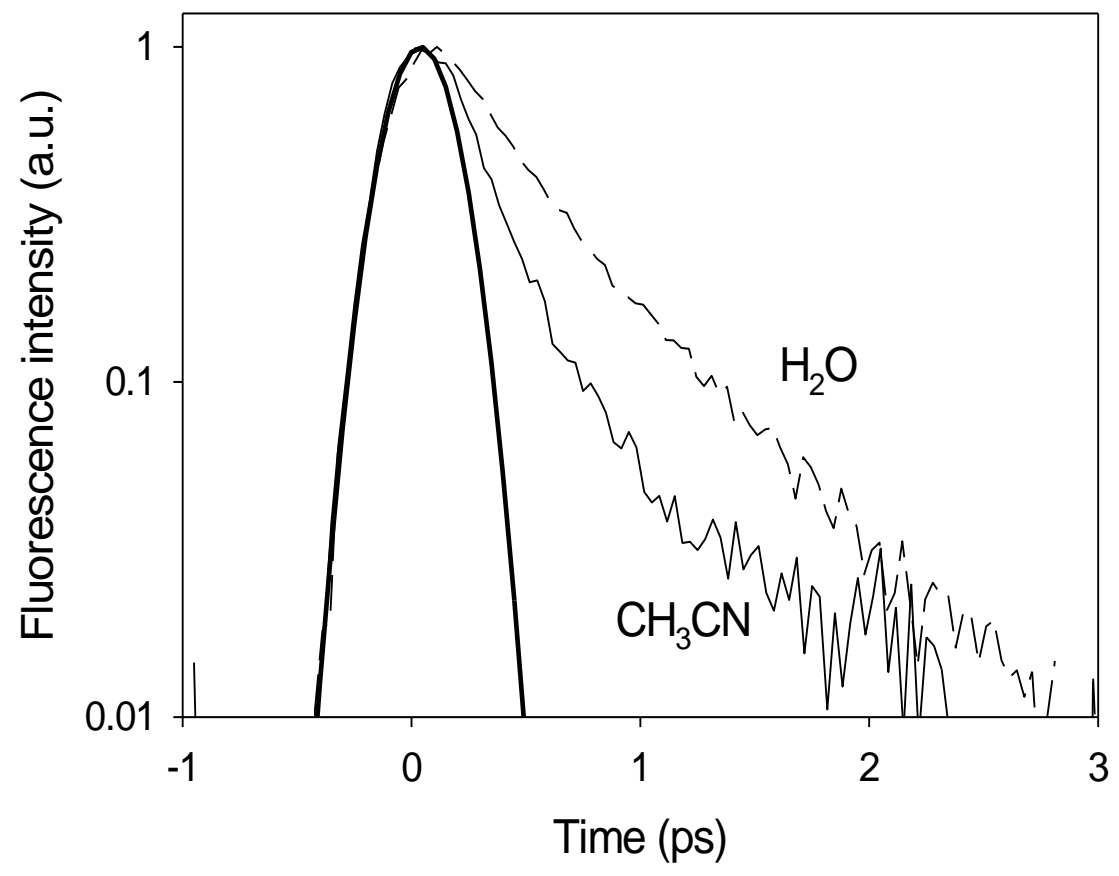

Figure 3. Fluorescence decays at $330 \mathrm{~nm}$ after excitation at $267 \mathrm{~nm}$ of thymine in $\mathrm{CH}_{3} \mathrm{CN}$ and water. Also shown (thick solid line) is the $330 \mathrm{fs}$ (fwhm) Gaussian apparatus function.

Our recordings with parallel and perpendicular excitation/detection configuration allowed us also to determine the fluorescence anisotropy $r(t)$ 


$$
r(t)=\frac{I_{p a r}(t)-G I_{\text {perp }}(t)}{F(t)}
$$

It was found that all zero-time anisotropies $\mathrm{r}_{0}$ are close to 0.4 , indicating no change in electronic structure between the absorbing and the emitting state.

Additional recordings of fluorescence decays were also made at $350 \mathrm{~nm}$, i.e. in the red wing of the fluorescence band. Only for thymine and 5-fluorouracil, showing bi-exponential decays, can any useful comparison be made with the recordings at $330 \mathrm{~nm}$. The lifetimes at 330 and $350 \mathrm{~nm}$ are identical within the experimental uncertainties.

\section{Discussion}

In this letter we have reported a detailed analysis of the excited state behavior of 6 uracils derivatives in acetonitrile. All the compounds examined exhibit an ultrafast excited state decay, in the sub picosecond range. Four of them, namely U, 6MU, 13DMU and TFT, have excited state lifetimes too fast ( $<100 \mathrm{fs}$ ) to be characterized by the experimental setup. Only the two 5-substituted compounds thymine and 5-fluorouracil show more complex behavior than uracil, exhibiting longer excited-state lifetimes and biexponential fluorescence decays. This picture is very similar to that obtained in water [17], suggesting that the intramolecular mechanism of the ground state recovery (v. infra) is the same in these two solvents.

On the other hand, the quantitative comparison between the results of the fluorescence upconversion experiments performed in acetonitrile and in water solution indicate that the solvent can significantly modulate the excited state lifetime of nucleobases. In fact, for three of the six compounds examined (namely thymine, 5-fluouracil and 5-trifluorothymine) the fluorescence lifetimes are noticeably shorter in acetonitrile than in water. The lifetimes of the remaining three compounds (U, 6MU, 13DMU) are instead too fast $(<100 \mathrm{fs})$, both in acetonitrile and water, to be characterized by our setup and thus no comparison is possible.

In particular for thymine our measurements in acetonitrile show a bi-exponential fluorescence decay, with an average lifetime of $235 \mathrm{fs}$, which is faster than that observed in water (388 fs). The fluorescence decay is dominated by an ultrafast component of $190 \mathrm{fs}$ (95\%) and complemented by a relatively slow component of $1.1 \mathrm{ps}$. On this basis it is possible to explain the disagreement between our results and those obtained by Häupl et al. [31], which measured a longer fluorescence lifetime of thymine in acetonitrile (2.4 ps) than in 
water (1.5 ps). We indeed believe that this difference is due to the low time resolution of the study of Häupl et al., i.e. they observe only the long tail of the fluorescence decay.

On the balance the results obtained in acetonitrile can be usefully interpreted within the same mechanistic framework we have developed in our previous studies in aqueous solution [17,32]. Confirming the results obtained by Matsika in the gas phase [18,19], the key step leading to the conical intersection between the ground $\left(\mathrm{S}_{0}\right)$ and the $\pi / \pi^{*}$ excited state $\left(\mathrm{S}_{\pi}\right)$ consists of the pyramidalization of the $\mathrm{C} 5$ carbon atom and in the out of the plane motion of the C5 substituent. Our calculations in aqueous solution indicates that this geometry rearrangement for thymine and, especially, for 5-fluorouracil should be more difficult than for uracil, giving account of the ordering of the excited-state lifetime [17]. The trend found in acetonitrile is the same: 5-fluorouracil exhibits the slowest excited state decay, followed by thymine. This supports our analysis of the intramolecular chemico-physical effect modulating the ground state recovery in uracils and suggests that solvent does not change the mechanism of the $S_{0} / S_{\pi}$ coupling.

It is thus necessary to explain how solvent modulates the excited state lifetimes of uracils. On this respect, the most significant finding we report in this letter is that also for thymine and 5TFT the fluorescence lifetime in $\mathrm{CH}_{3} \mathrm{CN}$ is shorter than that measured in water, even if the decrease is smaller than that found for 5FU. This result is consistent with our hypothesis that solvent affects the $S_{\pi}$ lifetime mainly by modulating the relative energy of the two lowest energy excited states in the Franck Condon region. Several theoretical studies indicate that in uracil derivatives a dark $n / \pi^{*}$ excited state $\left(S_{n}\right)$ has a stability very similar to that of the $S_{\pi}$ absorbing state $[1,17]$. Calculations including solvent effects have shown that the relative ordering of the $S_{n}$ and $S_{\pi}$ states may be reversed between the gas phase (or a non-polar aprotic solvent) and a polar, protic solvent like water [20]. In fact the stability of $S_{\pi}$ increases with the polarity and, most of all, the hydrogen bonding ability of the embedding medium. As a consequence, while in the gas phase this latter state is significantly less stable than $S_{n}$, in aqueous solution the situation is completely reversed. In acetonitrile solution, finally the two states have comparable stability in a wide portion of the PES in the proximity of the FC region [32]. In this latter solvent the presence of a planar $\mathrm{S}_{\mathrm{n}} / \mathrm{S}_{\pi} \mathrm{CI}\left(\mathrm{CI}^{\mathrm{n} / \pi}\right)$ in the proximity of the FC region is likely, constituting another effective decay channel for the wave packet on $S_{\pi}$ and a leading to a decrease of its experimental lifetime. It is indeed plausible that at the CI a part of the wave packet continues its motion on the $S_{n}$ surfaces towards the $S_{n}$ minimum, that in acetonitrile has a comparable stability to the $S_{n}$ one. Once reached the minimum of the $S_{n}$ 
dark state the system can decay non radiatively to the $S_{0}$ state. This extra decay channel should not be available in aqueous solution: at the $\mathrm{CI}^{\mathrm{n} / \pi}$ geometry $\mathrm{S}_{\mathrm{n}}$ is significantly less stable than $S_{\pi}$, due to the presence of the hydrogen bonded water molecules. The relative stability of the $S_{n}$ and $S_{\pi}$ states is similar for thymine and 5-fluorouracil; the above picture, originally developed for 5-fluorouracil [32] should thus be valid also for thymine. It cannot be excluded that the $\mathrm{CI}^{\mathrm{n} / \pi}$ decay channel is operative also for uracil and the other compound examined. For these latter compounds, however, the experimental fluorescence was at the limit of the apparatus sensitivity already in water. As a consequence, not only any comparison between the behavior in acetonitrile and in water is more difficult but the availability of another possible excited state decay route is also less important.

Further work is evidently necessary to judge the role of the solvent in this process and we are currently extending our ultrafast fluorescence studies to other bases and other solvent environments. Additional chemical-physical effects could indeed be involved in the solvent dependence of the excited state lifetime. For example, the solvent could modulate the barrier heights on the path towards the $\mathrm{CI} \mathrm{S}_{1} / \mathrm{S}_{0}$ conical intersection, even if preliminary calculations suggest that this should not be a major factor [32]. Dynamical computational studies should also be highly desirable. Still, at this point we note that it is possible to obtain a clear and consistent picture of the excited state decay of different nucleobases in different solvents by systematicly comparing experimental data with computational results $[17,32]$.

\section{Acknowledgements}

This study was financially supported by the CNRS within the framework of the European CERC3 program "Photochemistry of Nucleic Acids". 


\section{References}

[1] C. E. Crespo-Hernandez, B. Cohen, P. M. Hare, B. Kohler, Chem. Rev. 104 (2004) 1977

[2] J.-M. L. Pecourt, J. Peon, B. Kohler, J. Am. Chem. Soc. 122 (2000) 9348.

[3] J.-M. L. Pecourt, J. Peon, B. Kohler, J. Am. Chem. Soc. 123 (2001) 10370.

[4] B. Cohen, P. M. Hare, B. Kohler, J. Am. Chem. Soc. 125 (2003) 13594.

[5] R. J. Malone, A. M. Miller, B. Kohler, Photochem. Photobiol. 77 (2003) 158.

[6] B. Cohen, C. E. Crespo-Hernandez, B. Kohler, Faraday Discussions of the Chemical Society 127 (2004) 137.

[7] C. E. Crespo-Hernández, B. Kohler, J. Phys. Chem. B 108 (2004) 11182.

[8] L. Blancafort, B. Cohen, P. M. Hare, B. Kohler, M. A. Robb, J. Phys. Chem. A 109 (2005) 4431 .

[9] C. E. Crespo-Hernández, B. Cohen, B. Kohler, Nature 436 (2005) 1141.

[10] J. Peon, A. H. Zewail, Chem. Phys. Lett. 348 (2001) 255.

[11] T. Pancur, N. K. Schwalb, F. Renth, F. Temps, Chem. Phys. 313 (2005) 199.

[12] T. Gustavsson, A. Sharonov, D. Markovitsi, Chem. Phys. Lett. 351 (2002) 195.

[13] T. Gustavsson, A. Sharonov, D. Onidas, D. Markovitsi, Chem. Phys. Lett. 356 (2002) 49.

[14] D. Onidas, D. Markovitsi, S. Marguet, A. Sharonov, T. Gustavsson, J. Phys. Chem. B $106(2002) 11367$.

[15] A. Sharonov, T. Gustavsson, V. Carré, E. Renault, D. Markovitsi, Chem. Phys. Lett. 380 (2003) 173.

[16] A. Sharonov, T. Gustavsson, S. Marguet, D. Markovitsi, Photochem. Photobiol. Sci. 2 (2003) 1.

[17] T. Gustavsson, A. Banyasz, E. Lazzarotto, D. Markovitsi, G. Scalmani, M. J. Frisch, V. Barone, R. Improta, J. Am. Chem. Soc. 128 (2006) 607.

[18] S. Matsika, J. Phys. Chem. A 108 (2004) 7584.

[19] S. Matsika, J. Phys. Chem. A 109 (2005) 7538.

[20] R. Improta, V. Barone, J. Am. Chem. Soc. 126 (2004) 14320.

[21] M. Z. Zgierski, S. Patchkovskii, T. Fujiwara, E. C. Lim, J. Phys. Chem. A 109 (2005) 9384

[22] N. Ismail, L. Blancafort, M. Olivucci, B. Kohler, M. A. Robb, J. Am. Chem. Soc. 124 (2002) 6818.

[23] L. Blancafort, M. A. Robb, J. Phys. Chem. A 108 (2004) 10609. 
[24] M. Z. Zgierski, S. Patchkovskii, E. C. Lim, J. Chem. Phys. 123 (2005) 081101.

[25] C. M. Marian, J. Chem. Phys. 122 (2005) 104314.

[26] C. Marian, D. Nolting, R. Weinkauf, Phys. Chem. Chem. Phys. 7 (2005) 3306

[27] S. B. Nielsen, Theis I. Sølling, ChemPhysChem 6 (2005) 1276.

[28] S. Perun, A. L. Sobolewski, W. Domcke, Chem. Phys. 313 (2005) 107.

[29] S. Perun, A. L. Sobolewski, W. Domcke, J. Am. Chem. Soc. 127 (2005) 6257

[30] C. Canuel, M. Mons, F. Piuzzi, B. Tardivel, I. Dimicoli, M. Elhanine, J. Chem. Phys. 122 (2005) 0743161.

[31] T. Häupl, C. Windolph, T. Jochum, O. Brede, R. Hermann, Chem. Phys. Lett. 280 (1997) 520.

[32] T. Gustavsson, N. Sarkar, E. Lazzarotto, D. Markovitsi, V. Barone, R. Improta, J. Phys. Chem. B in press (2006).

[33] D. Markovitsi, D. Onidas, F. Talbot, S. Marguet, T. Gustavsson, E. Lazzarotto, J. Photochem. Photobiol. A: Chemistry in press (2006).

[34] L. B. Clark, G. G. Peschel, I. Tinoco, J. Phys. Chem. 69 (1965) 3615

[35] L. B. Clark, I. Tinoco Jr., J. Am. Chem. Soc. 87 (1965) 11. 